\title{
Perfil e padrão de uso de CRACK de Crianças e adolescentes EM SITUAÇÃO DE RUA: UMA REVISÃO INTEGRATIVA
}

\author{
Heloísa Garcia Claro ${ }^{1}$ \\ Márcia Aparecida Ferreira de Oliveira ${ }^{2}$ \\ Anna Paula Rocha Ribeiro ${ }^{3}$ \\ Caty Cilene Fernandes ${ }^{4}$ \\ Alexandre Silva Cruz 5 \\ Eva Geslaine Medina dos Santos ${ }^{6}$
}

Foi realizada revisão integrativa da literatura para reunir informações sobre o perfil e padrão de uso de crack de crianças e adolescentes em situação de rua. Foram selecionados 19 artigos em bases internacionais, publicados entre os anos 2000 e 2010. Encontrou-se que o uso é de padrão prejudicial, frequente, principalmente em famílias disfuncionais, em fragilidade social, em contextos de violência e exclusão, utilizando-se múltiplas drogas e sofrendo agravos, destacando-se doenças sexualmente transmissíveis, lesões associadas à violência, problemas respiratórios e cardiovasculares, além de implicações psicossociais. Esse quadro demanda ações intersetoriais, ainda escassas na realidade internacional, nas áreas de educação, saúde, segurança e assistência, para a implementação de estratégias de prevenção e tratamento do uso de crack e complicações.

Descritores: Cocaína Crack; Menores de Rua; Criança; Adolescente.

\footnotetext{
${ }^{1}$ Doutoranda, Escola de Enfermagem, Universidade de São Paulo, São Paulo, SP, Brasil.

${ }^{2} \mathrm{PhD}$, Professor Associado, Escola de Enfermagem, Universidade de São Paulo, São Paulo, SP, Brasil.

${ }^{3}$ Enfermeira, Prefeitura Municipal de São Paulo, São Paulo, SP, Brasil.

${ }^{4}$ Terapeuta Ocupacional, Prefeitura Municipal de São Paulo, São Paulo, SP, Brasil.

${ }^{5}$ Psicólogo, Prefeitura Municipal de Bertioga, Bertioga, SP, Brasil.

${ }^{6}$ Psicóloga, Prefeitura Municipal de Guarulhos, Guarulhos, SP, Brasil.
} 


\section{Profile ANd Pattern of CRACK USE By CHILdREN AND AdOLESCENTS LiVING ON THE STREETS: AN INTEGRATIVE REVIEW}

It was performed an integrative literature review to gather information about the profile and standard of crack use by children and adolescents living on the streets. 19 articles in international databases were selected, published between 2000-2010. It was found that the use is a harmful pattern, especially frequent in dysfunctional families, in social fragility, in contexts of violence and exclusion, where are used multiple drugs and suffer injuries, especially sexually transmitted diseases, injuries associated with violence, respiratory and cardiovascular problems, and psychosocial implications. This scenario requires intersectoral action, still scarce in the international situation, in the areas of education, health, security and assistance for the implementation of strategies for prevention and treatment of crack and complications.

Descriptors: Crack Cocaine; Homeless Youth; Child; Adolescent.

\section{PERFIL Y PADRón de USO de CRACK DE NIÑOS Y ADOLESCENTES EN SITUACIÓN DE CALLE: UNA REVISIÓN INTEGRATIVA}

Fue realizada una revisión integrativa de la literatura para reunir informaciones sobre el perfil y calidad de uso de crack de niños y adolescentes en situación de calle. Fueron seleccionados 19 artículos en bases internacionales, publicados entre los años de 2000 a 2010. Se encontró que el uso es de calidad prejudicial, frecuente principalmente en familias disfuncionales, en fragilidad social, en contextos de violencia y exclusión, que utiliza diversas drogas y sufre agravios, destacándose, enfermedades sexualmente transmisibles, lesiones asociadas a la violencia, problemas respiratorios y cardiovasculares, además de implicaciones psicosociales. Éste cuadro demanda acciones intersectoriales, aún escasas en la realidad internacional, en las áreas de educación, salud, seguridad y asistencia, para la implementación de estrategias de prevención y tratamiento del uso de crack y complicaciones.

Descriptores: Cocaína Crack; Jóvenes sin Hogar, Niño; Adolescente.

\section{Introdução}

Os estudos que foram realizados até o início da década de 80 , do século passado, não relatavam consumo alarmante de drogas entre crianças e adolescentes. No entanto, levantamentos realizados a partir de 1987 têm documentado tendência ao crescimento do consumo de diversas classes de drogas por essa população. Esses levantamentos foram realizados entre estudantes de primeiro e segundo graus, em dez capitais brasileiras, e também em amostras de adolescentes internados e entre meninos de rua. Em 1997, um desses estudos evidenciou tendência ao aumento do consumo dos inalantes, da maconha, da cocaína e de crack (substância derivada da cocaína), em determinadas capitais ${ }^{(1)}$.

O consumo de crack é considerado como um fenômeno em ascensão nos últimos anos, com graves repercussões a contingente importante de crianças e adolescentes, vindo a se configurar como mercado seguro entre as populações de baixa renda das periferias das grandes cidades, sendo esse fenômeno ainda mais expressivo entre meninos e meninas em situação de rua, um grupo excluído da sociedade, com pouca ou nenhuma assistência e de alto risco aos agravos à saúde integral de seus membros ${ }^{(2)}$. 
Em um estudo realizado com a população de crianças e adolescentes, foram investigados 21 indivíduos na faixa etária entre 11 e 17 anos. Por meio da análise dos dados, pode-se perceber predomínio do sexo masculino $(85,7 \%)$ na amostra total. O início do consumo de drogas ocorreu por volta dos 11 anos, sendo a porta de entrada o uso de álcool, caminhando rapidamente para tabaco, maconha, solventes, opioides, cocaína inalada e finalmente o crack, por volta dos 14 anos. As drogas mais consumidas foram o álcool, maconha, cocaína inalada, solventes e crack, nessa ordem, dados esses que diferem do encontrado em levantamentos nacionais que apontam drogas como solventes, maconha, anticolinérgicos e cocaína como as drogas mais consumidas nessa população $0^{(3)}$.

Do total de pacientes estudados, $57,1 \%$ usavam crack e mais da metade desses indivíduos fazia uso regular da droga. Esses usuários apresentavam, comparativamente ao grupo de usuários de outras drogas, evidências de maiores prejuízos escolares, envolvimento em atividades ilegais, assim como problemas em suas redes sociais (sobretudo com suas famílias) mais acentuados. Diferente do encontrado para as outras drogas, o uso regular de crack está associado à ocorrência de problemas em 100\% dos casos. Neste estudo, concluiu-se que o uso de crack surgiu nos estágios finais de consumo de drogas, com problemas de maior gravidade, especialmente em relação aos estudos e relações familiares, assim como o envolvimento em atividades ilegais ${ }^{(3)}$.

A cocaína e, sobretudo, o crack são drogas que podem desenvolver dependência de forma rápida. Assim, atividades ilícitas podem constituir o modo pelo qual crianças e adolescentes que não têm meios próprios adquiram as drogas ${ }^{(1)}$.

Apesar do acima exposto, dados sobre o perfil e padrão de uso de crack de adolescentes, em situação de rua, ainda são escassos na literatura nacional. Maiores informações acerca desses tópicos podem subsidiar ações futuras para enfrentamento dessa problemática, o que justifica estudos que caminhem nesse sentido.

\section{Objetivos}

Neste estudo objetivou-se realizar revisão integrativa da literatura nacional e internacional acerca do perfil e padrão de uso de crack por crianças e adolescentes em situação de rua.

\section{Métodos}

A revisão bibliográfica é um tipo de estudo que permite que o conhecido sobre um determinado assunto seja identificado, analisado e apresentado, de forma sucinta, ressaltando sua relevância. A síntese do conhecimento se faz indispensável para sua aplicabilidade no campo da prática $^{(4)}$.

Uma revisão bibliográfica do tipo integrativa permite que a literatura seja analisada de forma ampla, englobando estudos experimentais e não experimentais. Os autores pretendem, por meio deste estudo, apresentar dados relacionados ao perfil e padrão de uso de crack de crianças e adolescentes em situação de rua.

A revisão integrativa tem como finalidade reunir e sintetizar o conhecimento já produzido sobre o tema investigado, ou seja, permite buscar, avaliar e sintetizar as evidências disponíveis para a sua incorporação na prática.

Esse tipo de revisão possibilita uma análise da literatura que se faz útil para o meio científico, trazendo contribuições para futuras discussões, identificando e apontando novas necessidades para outras pesquisas na área. O conhecimento gerado por meio da revisão integrativa pode ser utilizado no campo das políticas de saúde, embasando a prática baseada em evidências ${ }^{(5-6)}$. Neste estudo, a revisão integrativa foi realizada em seis etapas, descritas a seguir.

\section{Primeira etapa (tema e questão norteadora)}

Por meio de uma análise preliminar da literatura, percebeu-se grande referência a crianças e adolescentes em situação de rua usuários de crack. Buscando fomentar pesquisas e intervenções focadas nessa população, buscaram-se informações a respeito das necessidades dessa população, o que incitou o seguinte questionamento: Qual é o perfil e padrão de uso de crack de crianças e adolescentes em situação de rua, descrito pela literatura nacional e internacional?

\section{Segunda etapa (busca bibliográfica)}

Foi realizada uma busca na literatura, e foram selecionadas 19 obras publicadas no período de 2000 a 2010, em idioma português e inglês, disponíveis nas bases de dados: Literatura Latino-Americana e do Caribe em Ciências da Saúde (LILACS), Medical Literature Analysis and Retrieval Sistem on-line (Medline), Scientific Eletronic Library Online (SciELO) e US National Library of Medicine-Nacional Institutes of Health (PubMed). Foram utilizados os seguintes Descritores em Ciência da Saúde (DeCS): criança de rua, adolescente, crack e cocaína.

\section{Terceira etapa (categorização)}

As informações dos artigos consideradas relevantes aos objetivos da pesquisa foram reunidas em fichas, para facilitar a análise dos dados. Essas fichas possuíam campos para preenchimento de informações como: informações acerca de dados sociodemográficos, informações relacionadas à família e rede social, padrão de uso, instituições que os usuários frequentam ou frequentaram, entre outras consideradas importantes para se atingir o objetivo deste estudo.

\section{Quarta etapa (avaliação dos estudos)}

As publicações selecionadas e catalogadas foram lidas na íntegra, e suas respectivas fichas foram submetidas a uma releitura para garantir que as informações relevantes dos artigos estivessem presentes nas mesmas. 
Quinta e sexta etapas (sintese do conhecimento e interpretação e discussão)

Nesta etapa os dados foram submetidos a uma análise comparativa, tendo sido organizados em quadros, facilitando para o leitor a avaliação dos resultados, sintetizando o conhecimento adquirido e tornando-o acessível $^{(7)}$. Por meio da análise dos quadros, foi possível levantar informações relevantes acerca do perfil e padrão de uso de crack por crianças e adolescentes em situação de rua, apresentadas na seção a seguir.

\section{Resultados}

Até aproximadamente o final da década de 90, do século passado, os estudos referem que o perfil predominante de usuários de crack era de homens em situação de pobreza, com idade inferior a 30 anos, desempregados, em sua maioria desabrigados e semianalfabetos. Por meio da análise dos artigos, percebe-se que esse consumo ainda predomina, na atualidade, em classes sociais mais baixas, porém, nos últimos dez anos, indivíduos de classes sociais mais elevadas têm iniciado progressivamente o uso dessa droga, provocando maior preocupação da sociedade acerca desse tema. As crianças e adolescentes brasileiros em situação de rua começaram a usar o crack no final dos anos 80 , do século passado, sobretudo nas Regiões Sul e Sudeste. Houve aumento nesse consumo, evidenciado em levantamentos realizados em 1987, 1989, 1993, 1997 e 2003. Esse aumento de consumo de crack é encontrado em diversos textos da literatura nacional ${ }^{(8)}$.

Os usuários de crack, em sua maioria, fazem uso de múltiplas drogas, com início precoce das substâncias psicoativas ilícitas. Indivíduos com histórico familiar de dependência ou abuso de drogas (em especial o álcool) apresentam maior tendência ao consumo de crack. Entretanto, em estudo que se utilizaram métodos estatísticos para associar variáveis ao consumo de crack, curiosamente encontrou-se que indivíduos que possuíam familiares com problemas associados ao uso de crack eram menos propensos a utilizar essa substância, uma vez que experenciaram graves consequências decorrentes desse uso ${ }^{(9)}$.

Após a experimentação de drogas lícitas, especialmente o álcool, esses indivíduos realizaram uso de drogas ilícitas, chegando, finalmente, ao consumo de crack. A maconha é normalmente a primeira droga ilícita da qual fazem uso. Encontra-se referência que esses indivíduos, em sua maioria, fizeram também uso anterior de cocaína injetável e intranasal. Quanto ao padrão de uso, a maior parte dos indivíduos utiliza a droga diariamente, em quantidades suficientes para considerá-los usuários "pesados" de crack. Encontrou-se, também, que o padrão de uso de crack em indivíduos fora de qualquer tipo de tratamento é maior do que em indivíduos em tratamento (não importando a metodologia empregada para tanto $)^{(8)}$. Um dos estudos realizou entrevistas com usuários e os entrevistados afirmaram ter controlado o uso de droga em certo momento, ou por não gostar dos efeitos das drogas ou por terem percebido que o uso de droga era prejudicial à situação deles, sobretudo no que se refere ao envolvimento em atividades ilícitas. Esses entrevistados simplesmente decidiram parar de usar droga e o conseguiram sem nenhuma intervenção profissional. Quando o suprimento da droga não estava disponível, eles referiram não ter nenhum problema por não usar. Nenhum dos entrevistados, neste estudo, parecia considerar o uso de droga como simplesmente positivo, sem nenhuma consequência negativa. Todos eles estavam conscientes de que o uso de droga era prejudicial, seja fisicamente, ou em relação ao seu comportamento. Para muitos, entretanto, o uso de droga continuava a ter papel importante na condição de tentar e conseguir viver nas ruas ${ }^{(10)}$.

Esses indivíduos fazem também uso de drogas injetáveis, contribuindo para a transmissão de diversas patologias. É presente também o relato de ocorrência de gravidez não planejada, decorrente de relações sexuais desprotegidas $^{(8-9,11-13)}$.

Como dito anteriormente, o uso de crack é relatado pela literatura como predominante em situações de empobrecimento social, entre indivíduos em situação de diversos riscos psicossociais, estando amplamente presente em contextos de moradia precária ou desabrigamento. Considerando esse contexto, encontraram-se citações acerca de organizações não governamentais que assistem esses indivíduos de alguma maneira, sendo essas importante fonte de apoio social para eles. Muitas vezes, os indivíduos iniciam o uso de crack como uma forma de ritual de iniciação nos grupos. Sem esse uso, os indivíduos frequentemente (principalmente as crianças e adolescentes) não são aceitos nos grupos, que são importantes para facilitar a sua sobrevivência no ambiente das ruas ${ }^{(9,14)}$.

$\mathrm{O}$ ambiente no qual vive o indivíduo é considerado como fator principal na jornada de desenvolvimento emocional desse, sendo que a maturidade completa do individuo não é possível no ambiente social imaturo e doente. Nesses locais, o objeto droga se apresenta como uma resposta às falhas de provisão ambiental e social ${ }^{(15)}$.

Em artigos nacionais e internacionais encontra-se referência de acúmulo de locais de compra e uso de crack nas regiões centrais das grandes cidades, locais esses com grande número de população em situação de rua ${ }^{(8-11,14)}$.

A totalidade dos artigos fez referência a vínculos familiares e sociais altamente empobrecidos. Os indivíduos relatam que deixam suas casas em decorrência da miséria econômica e afetiva, abuso físico e psicológico. Foi evidenciado, em alguns artigos, que no uso de substâncias psicoativas encontra-se a esperança de afastamento do ambiente perturbador e fragmentado, onde não existem espaços afetivos. Em alguns estudos encontraram-se dados que sugerem que usuários de crack possuem relações sociais mais pobres do que os usuários de outras drogas, sendo que esses indivíduos pertencem, em sua maioria, a famílias disfuncionais, onde é comum atos de violência verbal e física. Ressalta-se, também, o frequente relato de abuso sexual sofrido por esses indivíduos, contribuindo para o abandono do lar e/ou família. As relações mais fortes desses indivíduos são 
decorrentes do uso de drogas, uma vez que há referência a vínculos com outros usuários do crack, ações coletivas para conseguir a droga (os indivíduos se unem para execução de atividades ilícitas), como também vínculos com os fornecedores das drogas ${ }^{(2,9)}$.

Como fonte de financiamento para o uso, encontrouse, em especial, referências a atividades ilícitas, como pequenos roubos e furtos, prostituição e também participação em atividades relacionadas ao tráfico de drogas. No âmbito da exploração sexual infantil, as drogas são mencionadas no processo de iniciação das meninas na prostituição, como auxílio e forma de redução (ou adequação) do risco a valores aceitáveis com relação à sua situação de opressão e sofrimento. Esses indivíduos executam também atividades informais, como vendedores ambulantes, guardadores de carros, "flanelinhas", pedintes. Há forte referência ao abandono das atividades escolares, contribuindo para a baixa escolaridade dessa população ${ }^{(8,16-18)}$.

Há relato de "trocas" por drogas ou alimentos envolvendo favores sexuais. Para os meninos, as relações homossexuais, quando no papel passivo, são sinais de fraqueza e falta de masculinidade, não tendo caráter transitório. Diferentemente para as meninas, para as quais são experiências circunstanciais. Embora não seja uma constante, a homossexualidade e a prostituição perpassam o universo desses meninos e meninas ${ }^{(13,19)}$.

A situação de saúde desses indivíduos é agravada, sobretudo, em decorrência de sexo desprotegido e violência, resultando em número alto de contaminações por doenças sexualmente transmissíveis. Um dos estudos apresenta o dado de que $70 \%$ dos usuários de crack estudados sofreram abuso sexual em algum momento da vida. Outro estudo realizado na cidade de São Paulo evidenciou casos de crianças e adolescentes que vivem na rua e sofrem violências sexuais cotidianamente, inclusive há relatos de violência por parte de policiais, assim como indivíduos que acabam se relacionando e trabalhando para pessoas que administram clientes para a prostituição ${ }^{(8-9,11-13,16)}$.

Estudos realizados nos EUA detectaram indícios de forte associação entre uso do crack e transações de sexo por drogas ou dinheiro. Esse tipo de negociação fez com que a procura por tratamento, por parte de usuários de crack, aumentasse nos últimos anos. Apesar de indícios do consumo crescente de crack, encontrou-se que poucos estudos têm sido desenvolvidos no sentido de entender esse uso potencialmente comprometedor. Em um recente levantamento, parece nítido o aumento do uso dessa substância, em todo o Brasil, com destaque para São Paulo e Rio de Janeiro. O crescimento do uso de crack é muito mais evidente em São Paulo do que nas outras cidades, havendo diferença importante na prevalência do uso dessa droga e das suas formas de administração nas diversas Regiões do País ${ }^{(14)}$.

Os estudos apresentam referências a comorbidades psiquiátricas diagnosticadas em indivíduos usuários de crack e múltiplas drogas, destacando-se o relato de tentativas de suicídio, sobretudo entre as usuárias de crack do sexo feminino. Ocorre também a associação de patologias do trato circulatório e respiratório, decorrentes do uso dessas substâncias ${ }^{(16,20-21)}$.

A procura por instituições de saúde por parte dos indivíduos estudados nesses artigos limita-se, principalmente, a intervenções pontuais em salas de emergência com o objetivo de desintoxicação. Não foram encontradas referências de ações ou intervenções de atenção primária com essa população nos artigos pesquisados. Em média, 70\% dos pacientes admitidos em hospitais para tratamentos de problemas relacionados ao uso de cocaína são usuários de crack. Um estudo realizado com aproximadamente 400 indivíduos usuários de crack, do município de Santos, apresentou o dado de que ao menos $20 \%$ desses relataram ao menos um episódio de overdose $^{(21)}$. Encontra-se, também, a sugestão de que as intervenções de profissionais da saúde caminhem no sentido da observação global, estabelecendo planos individuais de intervenção e, ainda, atenção em serviços especializados $^{(1)}$.

\section{Discussão}

Os resultados encontrados neste estudo são corroborados por estudos anteriores. Um exemplo disso é o fato de que alguns estudos concluem que o perfil sociodemográfico do usuário de crack sofreu discretas mudanças ao longo dos últimos 30 anos, sendo que o uso compulsivo é ainda majoritário, com importante comprometimento físico, moral e social do usuário. $\mathrm{O}$ uso exclusivo de crack foi aqui evidenciado como pouco presente, uma vez que, frequentemente, o mesmo é associado a outras drogas, caracterizando esses indivíduos como usuários de múltiplas drogas ${ }^{(22)}$.

A literatura sugere que os efeitos do crack estão relacionados aos prejuízos físicos psíquicos, sociais e financeiros, forçando os seus usuários a participar de atividades ilícitas, tornando-os vulneráveis a doenças infecciosas e mortes por causas externas relacionadas à violência decorrente dessas atividades.

As principais complicações do uso de crack relacionadas à saúde, levantadas neste este estudo, são a contaminação por doenças sexualmente transmissíveis, lesões decorrentes de abusos e violência, homicídio e episódios como overdose. Esse dado vai ao encontro do que foi levantado anteriormente, que mostra dados acerca de causas de morte relacionadas ao uso de crack listando, respectivamente, episódios de overdose, condições decorrentes da contaminação pelo vírus HIV e homicídios $^{(23)}$.

Pode-se afirmar também que os dados encontrados acerca da prostituição, como forma de conseguir a droga, são de extrema preocupação na literatura científica. De acordo com estudos anteriores, discussões, brigas, ameaças, roubos e tráfico de drogas representam a maior parte dos crimes e violências cometidos por usuários de crack. Esse dado também foi confirmado nos resultados aqui apresentados, mostrando prevalência de envolvimento em atividades ilegais para mobilizar recursos e conseguir a droga ${ }^{(24)}$. 
A mobilização e as diferentes maneiras de se conseguir a droga são temas de diversos estudos, merecendo atenção o ambiente que essas crianças e adolescentes vivem. Os usuários de crack buscam alívio para um cotidiano que é atravessado por diferentes formas de opressão e poucas perspectivas de mudança, o que está em consonância com os resultados apresentados em que o ambiente sociofamiliar é descrito como ambiente hostil, violento, de miséria e experiências negativas ${ }^{(25-26)}$.

A droga cria a capacidade do prazer imediato, o que diminui o prazer de se viver outros interesses, no qual se pode destacar o abandono dos estudos e poucos relatos sobre o brincar como forma de ocupação, dando lugar às exigências relacionadas a conseguir dinheiro como forma de sobrevivência e manutenção do uso de drogas, o que intensifica o processo de marginalização e exclusão dessas crianças e adolescentes ${ }^{(14)}$.

Apesar desse quadro preocupante de uso de crack, em destaque para as grandes cidades, ser comprovado pela literatura, este estudo evidenciou que as políticas de atendimento a esses usuários não aparecem implantadas e implementadas nessas metrópoles, o que representa descompasso entre a demanda e a oferta de atendimento, uma vez que são realizadas intervenções desarticuladas pelas organizações não governamentais, hospitais e outros equipamentos, sem projetos intersetoriais e, sobretudo, sem a articulação necessária da rede de atenção integral às pessoas usuárias de álcool e outras drogas.

O uso controlado de crack esteve presente em alguns estudos relacionados à própria vontade do usuário de parar com o uso, ou em trabalhos desenvolvidos pelas equipes de saúde, pelos consultórios de rua e por alguns serviços especializados. Esses foram destacados pelos usuários como positivos, porém, evidenciou-se, nos resultados ora apresentados, que não existem relatos de intervenções de atenção primária relacionadas ao uso de crack, que seriam de extrema importância para o estabelecimento de uma rede de atendimento a essa população. Consideradas em conjunto, as implicações associadas ao uso de crack consistem em importante problema de saúde pública, devido à gravidade dos problemas associados ao uso abusivo, sendo necessário o desenvolvimento de projetos de intervenção e políticas públicas ao seu controle, sendo de suma importância a união dos setores da educação, assistência social, segurança pública e saúde, bem como de iniciativas do terceiro setor, para que as intervenções se tornem de fato eficazes ${ }^{(27)}$.

Em relação à vulnerabilidade de crianças e adolescentes, principalmente em situação de rua, pouco se discutiu nos artigos pesquisados. Esse é um dado que se destaca, uma vez que é amplamente difundido na literatura que a adolescência é um período de transição, tanto física quanto emocional, tornando o adolescente vulnerável, sobretudo em relação às drogas, em decorrência das condições adversas apresentadas nessa fase, tais como a influência do grupo de amigos, busca pelo desconhecido, curiosidade, fuga das dificuldades, contradição dos valores estabelecidos pelos familiares entre outros ${ }^{(28)}$.

\section{Conclusões}

Este estudo objetivou reunir informações acerca do perfil e padrão de uso de crack por crianças e adolescentes em situação de rua. Por meio dos resultados levantados e da discussão da literatura científica, conclui-se que o uso de crack é altamente frequente nessa população, ocasionando agravos à saúde e sociais, no cotidiano desses indivíduos. Esses são, em sua maioria, indivíduos marginalizados, fora do sistema formal de educação e emprego, pertencentes a grupos que executam atividades ilegais para conseguir a droga, tornando-se cada vez mais vulneráveis e excluídos. Em sua maioria, utilizam a droga de maneira "abusiva", mesmo tendo consciência dos efeitos negativos da mesma, em associação ao uso de diversas outras drogas, o que os expõe, ainda, a mais riscos, pois a questão de maior preocupação no que concerne às drogas não é o seu uso, e sim o abuso, um padrão de consumo de substância que pode conduzir maiores prejuízos para o indivíduo.

Apesar de mais frequente entre a população das camadas sociais mais baixas (destacando os indivíduos em situação de rua), atinge atualmente a sociedade como um todo, seja em decorrência do consumo dessa droga, como também sofrendo os prejuízos sociais que dele decorrem e o reforçam.

Para lidar com esse problema, é necessário o envolvimento dos diversos setores da sociedade, de modo a organizar uma rede de atenção ao usuário de crack, ou seja, uma rede de atenção integral aos usuários de álcool e outras drogas. Essa rede deve ser capaz de incluir desde a atenção primária, para evitar o uso experimental da droga, até a desintoxicação dos usuários. Devem estar envolvidos nessas ações principalmente os setores de assistência social, educação, saúde e segurança, para garantir a plena reabilitação dos indivíduos que sofrem as consequências do consumo de drogas.

\section{Referências}

1. Marques ACPR, Cruz MS. O adolescente e o uso de drogas. Rev Bras Psiquiatr. 2000;22(1):32-6.

2. Muza GM, Bettiol H, Muccillo G, Barbieri MA. Consumo de substâncias psicoativas por adolescentes escolares de Ribeirão Preto, SP (Brasil). II Distribuição do consumo por classes sociais. Rev Saúde Pública. 1997;31(2):163-70.

3. Scivoletto S, Henriques SG Júnior, Andrade AG. Uso de drogas por adolescentes que buscam atendimento ambulatorial: comparaçäo entre "crack" e outras drogas ilícitas - um estudo piloto. Rev Assoc Bras Psiquiat. 1997;19(1):7-17.

4. Silveira RCCP. O cuidado de enfermagem e o cateter de Hickman: a busca de evidências. [Dissertação de Mestrado em Enfermagem]. Ribeirão Preto: Escola de Enfermagem de Ribeirão Preto da Universidade de São Paulo; 2005. 134 p.

5. Fernandes LM. Úlcera de pressão em pacientes críticos hospitalizados. Uma revisão integrativa da literatura. [Dissertação de Mestrado em Enfermagem] Ribeirão Preto: 
Escola de Enfermagem de Ribeirão Preto da Universidade de São Paulo; 2000. 168 p.

6. Whittemore R, Knafl K. The integrative review: updated methodology. J Adv Nurs. 2005;52(5)546-53.

7. Balieiro CRB. Características da violência doméstica relacionadas ao trauma por queimadura em crianças: Revisão integrativa da literatura. [Dissertação de Mestrado em Enfermagem]. Ribeirão Preto: Escola de Enfermagem de Ribeirão Preto da Universidade de São Paulo; 2009. 117p.

8. Duailibi LB, Ribeiro M, Laranjeira R. Profile of cocaine and crack users in Brazil: [review]. Cad Saúde Pública. 2008;24(supl.4):s545-s57.

9. Paquette C, Roy E, Petit G, Boivin JF. Predictors of crack cocaine initiation among Montreal street youth: A first look at the phenomenon. Drug Alcohol Depend. 2010;110(1-2):85-91.

10. Schenker D. Juventude desabrigada e abuso de drogas: pesquisando as necessidades dos meninos de rua em Salvador (Brasil). In: EDUFBA (BR). Drogas: tempos, lugares e olhares sobre seu consumo. [Internet]. 2004. [acesso 4 set 2011]. Disponível em: http://www.twiki.ufba. br/twiki/pub/CetadObserva/Adolescencia/Juventude desabrigada_e_abuso_de_drogas.pdf

11. Werb D, Kerr T, Fast D, Qi J, Montaner JSG, Wood E. Drug related riscs among street youth in two neighborhoods in a Canadian Setting. Health \& Place. 2010; 16(5):1061-7.

12. Frangella SM. Fragmentos de corpo e gênero entre meninos e meninas de rua. Cad Pagu. 2000;14:231-4.

13. Bellini LM, Nogueira LA. Sexualidade e violência, o que é isso para jovens que vivem na rua? Paraná. Texto Contexto Enferm. 2006;15(4):610-6.

14. Raupp L, Adorno RCF. Circuitos de uso de crack na região central de São Paulo. Ciênc Saúde Coletiva. 2011;16(5):2613-22.

15. Monteiro LFC. O uso de substâncias psicoativas por crianças e adolescentes em situação de rua: uma leitura winnicottiana. In: EDUFBA (BR). Toxicomanias: incidências clínicas e socioantropolicas [Internet]. EDUFBA;2009. [acesso 04 set 2011].p. 141-59. Disponível em: http:/www.twiki.ufba.br/twiki/pub/CetadObserva/ Adolescencia $/ \% 280$ uso de subst $\%$ e2ncias psicoativas_por_crian $\% \mathrm{e} 7 \mathrm{as} \_\mathrm{e} \_$adolescentes_em _ situa $\%$ e $7 \%$ e3o_de_rua.doc\% $\% 29$.pdf

16. Gomes R, Minayo MCS, Fontoura HA. A prostituição infantil sob a ótica da sociedade e da saúde. Rev Saúde Pública. 1999;33(2):171-9.

17. Campos TN, Del Prette ZAP, Del Prette A. Sobrevivendo nas Ruas: Habilidades Sociais e Valores de Crianças e Adolescentes. Psicol Reflexão e Critica. 2000;13(3):517-27.

18. Conceição MIG, Sudbrack MFO. Estudo sociométrico de uma instituição alternativa para crianças e adolescentes em situação de rua: construindo uma proposta pedagógica. Psicol Reflexão e Crítica. 2004;17(2):277-86.

19. Medeiros M, Ferriani MGC, Munari DB, Gomes R. The meaning of sexuality for adolescents living on the street in Goiânia. Rev. Latino-Am. Enfermagem. 2001;9(2):35-41.
20. Perri CP, Laranjeira RR, Silveira DX, Dunn J, Formigoni MLOS. Aumento da procura de tratamento por usuários de crack em dois ambulatórios na cidade de Säo Paulo, nos anos de 1990 a 1993. Rev Assoc Med Bras. 1997;43(1):25-8.

21. Pulcherio G, Stolf AR, Pettenon M, Fensterseifer DP, Kessler F. Crack - da pedra ao tratamento. Rev da AMRIGS. 2010;54(3):337-43.

22. Oliveira AG, Nappo AS. Caracterização da cultura de crack na cidade de São Paulo: padrão de uso controlado. Rev Saúde Pública. 2008;42(4):664-71.

23. Ribeiro M, Dunn J, Sesso R, Dias AC, Laranjeira R. Causes of death among crack cocaine users. Rev Bras Psiquiatr. 2006;28(3):196-202.

24. Carvalho HB, Seibel SD. Crack cocaine use and its relationship with violence and HIV. Clinics. 2009;64(9):857-66.

25. Raupp L, Adorno RCF. Uso de crack na cidade de São Paulo - Brasil. Rev Tóxicodependências. 2010; 16(2):29-37.

26. Bourgois P. In search of Horatio Alger. In: Reinarman C, Levine HG, editors. Crack in America: demon drugs and social justice [Internet]. London: University of California Press; 1997. [acesso 4 set 2011]. Disponível em: https://www.ncjrs.gov/App/Publications/abstract.aspx? $\mathrm{ID}=170651$

27. Oliveira AG, Nappo AS. Caracterização da cultura de crack na cidade de São Paulo: padrão de uso controlado. Rev Saúde Pública. 2008;42(4):664-71.

28. Lima IS, Magalhães PM, Zaleski EGF, Arantes SL. História oral de vida de adolescentes dependentes químicos internados no setor de psiquiatria do Hospital Regional de Mato Grosso do Sul para tratamento de desintoxicação. SMAD, Rev. Eletrônica Saúde Mental Álcool Drog. (Ed. port.). 2008;4(1):1-11. 